\title{
Dynamic Sexual Offense Risk Assessment Using the VRS- SO With Indeterminate Sentenced Men
}

\author{
Mark E. Olver ${ }^{1}$
}

[1] University of Saskatchewan, Saskatoon, SK, Canada.

Sexual Offending: Theory, Research, and Prevention, 2021, Vol. 16, Article e3745, https://doi.org/10.5964/sotrap.3745

Received: 2020-05-22 • Accepted: 2021-06-11 • Published (VoR): 2021-12-23

Handling Editor: Martin Rettenberger, Centre for Criminology (Kriminologische Zentralstelle - KrimZ), Wiesbaden, Germany

Corresponding Author: Mark E. Olver, Department of Psychology and Health Studies, 9 Campus Drive, Arts Building Room 154, Saskatoon, Saskatchewan, Canada S7N 5A5. E-mail: mark.olver@usask.ca

Related: This article is part of the SOTRAP Special Thematic Section "Dynamic Assessment of Men Facing Indeterminate Detention”, Guest Editors: Andrew J. R. Harris \& Mark E. Olver, Sexual Offending: Theory, Research, and Prevention, 16, https://doi.org/10.5964/sotrap.v16

\section{Abstract}

Indeterminate detention (ID) is a high stakes sanction reserved for exceptionally high risk-high need (HRHN) persons who are deemed to pose an undue risk to public safety. It is one of the most extreme measures that is routinely taken by justice systems to manage sexual violence risk and prevent sexual and violent recidivism. Naturally, risk assessment is most frequently employed as a mechanism to keep dangerous people in custody; but seldom is risk assessment viewed as a possible ticket out for men with an ID designation who have made substantive risk changes and whose risk can be safely managed in the community. This article features applications of a dynamic sexual violence risk assessment and treatment planning tool, the Violence Risk ScaleSexual Offense version (VRS-SO), with ID individuals and other HRHN men, to assess risk in a dynamic manner to inform risk management efforts and release decisions. VRS-SO data on an ID sample are presented along with clinical illustrations of dynamic risk assessment. Several propositions are made with supporting data from VRS-SO normative research with treated sexual offending samples regarding the use of dynamic tools with ID men and the perils and pitfalls of relying solely on static measures. Ultimately, dynamic risk instruments can be used to track progress and monitor risk change over multiple assessments to inform release and reintegration decisions with ID persons. In this regard, dynamic assessment has the potential to help, rather than hinder, reintegration of ID sentenced persons and can inform safe, fair, and humane decisions. 


\section{Keywords}

VRS-SO, sexual recidivism, dynamic, risk assessment, dangerous offender

\section{Non-Technical Summary}

\section{Background}

People in the criminal justice system who are viewed to pose an exceptional danger to society may be subjected to preventative measures, such as a period of incarceration or hospitalization with no foreseeable release date. These measures are referred to as indeterminate detention (ID).

\section{Why was the study done?}

For humanitarian and financial considerations, ID sentenced persons eventually have to be let out; but concerns for public safety and the potential for recidivism result in very low release rates. This article argues that having an evidence-based system in place to evaluate risk on changeable dimensions for ID persons who participate in treatment and learn to manage their risk, is feasible and desirable to promote safe re-entry and public safety.

\section{What did the researchers do and find?}

The Violence Risk Scale-Sexual Offense version (VRS-SO) is one possible tool to use with ID persons to assess risk on a repeated basis to evaluate when the individual's risk can be safely managed in the community and how this may be done. Supporting data are presented to model hypothetical reoffense rates of ID persons when they are released after participating in treatment and present some cautious optimism that: a) treatment can help lower risk for ID persons, and b) that instrumentation is in place to inform safe release decisions with this population.

\section{What do these findings mean?}

A dynamic approach to assessment has the potential to help, rather than hinder, release of ID persons back to society and can inform safe, fair, and humane decisions. 


\section{Highlights}

- In Canadian corrections, treating men who have committed sexual offenses who are high risk-high need (HRHN) and reintegrating them safely to the community has been standard practice for a long time.

- Men with Dangerous Offender (DO) designations from a small sample of men treated for sexual offending, were found to have similar sexual violence risk profiles as other high risk-high need men without indeterminate detention (ID) designations.

- DO sentenced men were found to make risk-relevant changes from preposttreatment; results from modelling hypothetical recidivism rates based on their risk profile and change, indicated concordant reductions in risk for future sexual violence that were not appreciably different from that of other treated HRHN men who are routinely released.

- Dynamic risk instruments can be used to track progress and monitor risk change over multiple assessments to inform release and reintegration decisions with ID persons.

Indeterminate detention (ID) is a high stakes sanction reserved for exceptionally high risk-high need (HRHN) persons who are deemed to pose an undue risk to public safety. It is one of the most extreme measures that is routinely taken by justice systems to manage sexual violence risk and prevent sexual and violent recidivism. In North America, ID can take different forms. A prominent example in the US is the sexually violent predator (or persons) statute (SVP). The specifics of the SVP statute vary among the 21 jurisdictions with this legislation, but a common thread is the imposition of an indefinite period of civil commitment post sentence expiry for persons convicted of sexual offenses who are essentially declared to be an unmanageable release risk and who have a mental disorder linked to their sexual offending (Prentky et al., 2006). As such, SVP programs in the US are sometimes referred to as civil commitment.

The Canadian proxy for SVP is the Dangerous Offender (DO) legislation (Sec. 753, CCC), the focus of the present article. DO legislation emerged initially in 1977 and over the years there have been various amendments in the application for DO designation and sentencing of DO cases. In contrast to SVP civil commitment, which occurs prior to the would-be expiration of the individual's custodial sentence, the DO designation is usually applied at the point of sentencing for certain designated sexual or violent offenses (termed "serious personal injury offence") for persons deemed to pose a substantial threat to public safety. The determination essentially involves a persistent pattern of serious violent and aggressive behavior and/or behavior that demonstrates "failure to control his or her sexual impulses", and an appraisal that the individual poses an exceptionally high risk for sexual or general violence. Until 2008, the DO designation automatically yielded an indeterminate sentence, but now judicial discretion exists to 
assign determinate sentences, with or without a period of long-term supervision to follow (Sec. 753 (4)(a-c)). DO designations have steadily increased in Canada over the past 15 years, a recent profile of which is provided by Public Safety Canada's 2017-2018 Corrections and Conditional Release Statistical Overview (Public Safety Canada, 2019). Since 1978, 921 individuals have been adjudicated DO and 792 remain under federal correctional jurisdiction; $90 \%$ of these cases were in custody, while the remaining $10 \%$ were supervised in the community. Of the current 712 DOs in Canada, most (81.3\%) are serving an indeterminate sentence, of whom $95.5 \%$ are in custody and $4.5 \%$ under community supervision. Indigenous persons currently comprise 35.5\% of DOs. Since 1978 about two thirds (67.9\%) of individuals with DO designations had at least one conviction for a sexual offense (Public Safety Canada, 2019); however, perhaps the most detailed examination of the DO population profile (Trevathan et al., 2002) examined 179 DOs admitted between 1994-2001, finding that 85\% had an index conviction for a sexual offense.

In short, men carrying ID sentences in Canada are most frequently DOs adjudicated for sexual offenses. They are serving long sentences, they have challenging release prospects, yet they participate in treatment and release planning, and eventually, are intended to be released and supervised in the community. Risk assessment is one mechanism used at the front end to inform sentencing decisions of DOs (e.g., indeterminate vs. determinate); but then risk is repeatedly reassessed, initially following the first 7 years of custody after a DO designation and then every two years thereafter. In other words, ID sentenced men, and DOs in particular, undergo a lot of risk assessments. While risk assessment may be viewed as a mechanism to keep "dangerous" people in custody, it can also be viewed as a promising release mechanism, for instance, for people who have "changed" and whose risk can be safely managed in the community. Arguably dynamic sexual violence risk assessment may be used to appraise risk, inform risk reduction case planning, reappraisals of risk, and inform community reintegration efforts.

\section{A Primer on the VRS-SO}

The Violence Risk Scale-Sexual Offense version (VRS-SO; Wong et al., 2003-2017) was developed in 1999-2000 in partnership between Correctional Service Canada (CSC) and University of Saskatchewan and revised in subsequent years. Comprised of seven static and 17 dynamic items, the VRS-SO is designed to assess risk for future sexual offending, identify criminogenic targets for intervention planning, assess readiness for treatment, and assess changes in risk that may be attributable to intervention or other change agents (e.g., aging, improvement in community supports). It is an empirical actuarial clinician rated measure, with items rated on the basis of file and interview, and items summed to generate risk scores that are: a) linked to sexual recidivism estimates and b) can be arranged into one of five possible risk levels (Level I through IVb) to aid risk communication. The Static-99R (Helmus et al., 2012) can be substituted in place of the 
VRS-SO's static items and integrated with dynamic item ratings to generate recidivism estimates and/or a risk level (Olver, Mundt, et al., 2018).

The VRS-SO's risk categories and recidivism estimates were generated through logistic regression modelling on four combined samples of incarcerated men with a history of sexual offending $(N=913)$, each of whom had pretreatment and posttreatment VRS-SO ratings and followed up a minimum of 10 years post-release in the community. Logistic regression was employed to generate 5 and 10-year estimates of sexual recidivism as a function of baseline risk (VRS-SO total score or Static-99R and VRS-SO dynamic score) and change (VRS-SO dynamic change score). Logistic functions were created for each model and an Excel workbook (Mundt, 2015) was created to compute recidivism estimates for specific combinations of risk and change scores (available online free at www.psynergy.ca). In turn, a five-level risk system based on indexes of relative (e.g., VRS-SO score percentiles) and absolute (i.e., recidivism estimates) risk was generated, with consultation to authoritative guidelines (Hanson et al., 2016) and applications elsewhere with existing tools, such as Static-99R (Hanson et al., 2017).

The structure and composition of the VRS-SO lends itself for use with ID sentenced men, and DOs in particular, for various reasons. For one, with a possible range of 0-72 for total scores and 0-51 for dynamic scores, the VRS-SO has both a low floor and a high ceiling. Maxing out scores on the tool is no light task (the highest score in the normative sample is 67), while individuals with extremely low scores are in turn likely very low risk (i.e., would have limited offense history and few or no substantive areas of risk and need). Second, the VRS-SO can track and measure changes in risk; specifically, the dynamic items include a structured metric of change via the stages of change model operationalized for each dynamic item (described in the Method in further detail). As such, changes in risk are assessed in a structured manner and applied in an actuarial manner to adjust risk estimates as warranted from treatment or other agents of change. As such, while the VRS-SO might be used to justify an ID designation at the front end, it can also be used to justify making empirically-based non-arbitrary release and reintegration decisions.

\section{Current Study and Rationale}

In this article I advance, through a series of five propositions, that dynamic risk assessment measures can and should be used with ID sentenced persons with a history of sexual offending, specifically, to assess dynamic sexual violence risk and to inform risk management efforts and release decisions. I focus specifically on the DO designation in Canada, given that this represents the population of persons with ID sentences, most of whom have current or previous convictions for sexual violence. The article extends the limited previous work examining profiles and release outcomes of DOs, drawing on applications of the VRS-SO with DO designated men serving ID sentences for sexual offenses compared to other HRHN men. VRS-SO data on a Canadian DO sample are 
presented with clinical illustrations of dynamic risk assessment with DOs or otherwise ID sentenced men. The findings and conclusions may also extend to US sexual offense civil commitment contexts, given the ID nature of the term of hospitalization owing to perceived dangerousness and the mandate to ultimately treat and reintegrate this population.

\section{Method}

The present study was part of a broader program of ongoing sexual violence risk assessment and change research-featuring applications of the VRS-SO with federally sentenced men convicted for sexual offenses-that received ethical approval from the University of Saskatchewan Research Ethics Board (Beh\#15-308) as well as operational approval from Correctional Service Canada (CSC).

\section{Sample}

The sample featured 43 men serving ID sentences for at least one contact sexual offense. The men averaged 43.8 years $(S D=10.1$, range 20.5 to 64.8$)$ at the time of program admission. In all, $65.1 \%(n=28)$, men were White and 30.2\% $(n=13)$ were Indigenous, and $2.3 \%(n=2)$ were of other ethnocultural descent. Victim demographic information was available for about three quarters of the sample $(n=31 / 43)$, of whom, most men $(64.5 \%$, $n=20$ ) were convicted for sexual offenses exclusively against an adult victim, while smaller equal portions $(16.1 \%, n=5)$ each had exclusively extrafamilial child victims or a mixed victim profile (i.e., both adults and children), and $n=1(3.2 \%)$ had intrafamilial child victims only. Most $(83.3 \%, 25 / 30)$ but not all men had a previous conviction for a sexual offense.

\section{Treatment Program}

Treatment information was available for over three quarters of the sample $(76.7 \%, 33 / 43)$. Those men who received services attended either the moderate or high intensity stream of the National Sex Offender Program (NaSOP) across CSC institutions or the Clearwater High Intensity Sex Offender Program (similar in form and content to high intensity NaSOP) in the Prairie Region of CSC. NaSOP was a modular multi-intensity sexual offense treatment program delivered to men with a current or previous history of sexual offending (Yates et al., 2000). The program was broadly cognitive behavioral based and subscribed to the risk-need-responsivity (RNR; Andrews \& Bonta, 2010) principles of effective correctional treatment. The program was subdivided into low (2-4 months), moderate (4-5 months) and high intensity (8-9 months) streams. It involved a combination of group and individual services with treatment foci that included addressing deviant sexual interests and problems with sexual self-regulation, attitudes and cognitions supportive of 
sexual violence, relationship and intimacy skills, and emotion management, among other areas. Ancillary services including cultural programming, educational upgrading, vocational retraining, substance management services and the like were routinely available at the men's parent institutions. The NaSOP was delivered by trained program facilitators whose activities were overseen by a registered psychologist.

\section{Measures}

\section{Static-99R}

Static-99R (Hanson \& Thornton, 1999, 2000; Helmus et al., 2012) is a 10-item static actuarial sexual violence risk assessment instrument. Item content includes sexual and nonsexual offense history and perpetrator and victim demographic characteristics. It is intended to be used with an adult male population with a formal criminal history of sexual offending. With possible scores ranging from -3 to 12 , Static-99R scores can be arranged into five risk bands corresponding to Very Low Level I (-3, -2), Below Average Level II (-1, 0), Average Level III (1-3), Above Average Level IVa (4, 5), and Well Above Average Level IVb (6-12) (Hanson et al., 2017). Static-99R is the most frequently used sexual offense risk tool in the field (Kelley et al., 2020). Results of meta-analyses support its predictive accuracy for sexual and violent recidivism (Helmus et al., 2012).

\section{VRS-SO}

The VRS-SO (Wong et al., 2003-2017) is a sexual offense risk assessment and treatment planning tool designed to appraise risk for future sexual offending, identify targets for treatment and risk management efforts, evaluate readiness to change, and to assess changes in risk from treatment or other change agents. The VRS-SO consists of seven static (historical, generally unchanging) and 17 dynamic (potentially changeable) items scored on a 4-point $(0,1,2,3)$ ordinal scale. Dynamic items receiving a 2 or 3 rating are considered to be criminogenic (i.e., linked to sexual offending and prioritized for treatment), while 0 and 1 ratings represent low risk items. Results of exploratory and confirmatory factor analyses of the VRS-SO dynamic items demonstrate that they can be organized into three oblique factors termed Sexual Deviance (e.g., deviant sexual preference, sexual compulsivity, sexually deviant lifestyle), Criminality (e.g., interpersonal aggression, substance abuse, impulsivity), and Treatment Responsivity (e.g., cognitive distortions, insight) (Beggs \& Grace, 2010; Olver \& Eher, 2020; Olver, Neumann, et al., 2018; Olver et al., 2007).

Change is evaluated via a modified application of the stages of change (SoC) model which represents cognitive, behavioral, and experiential changes an individual makes as they attempt to ameliorate problem areas (Prochaska et al., 1992). Five stages are operationalized for each dynamic item along a continuum (i.e., Precontemplation, Contemplation, Preparation, Action, and Maintenance). Progression entails a greater awareness of one's problem area and increase in the frequency and duration of skills and 
strategies used to manage and replace problem behaviors. Criminogenic dynamic items (i.e., receiving a 2 or 3 rating) are given a baseline rating at pretreatment and then the SoC is rerated posttreatment (or subsequent assessment interval); 0- or 1-rated items do not generally receive an SoC rating since they do not represent problem areas in need of intervention. Progression from one stage to the next, in the direction of risk reduction corresponds to a 0.5 deduction in score, 2 stages, a 1-point deduction and so on; the lone exception is movement from Precontemplation to Contemplation since this does not entail behavioral change. This is done for each item identified as criminogenic at baseline; change ratings across the items are then summed to generate a total change score.

VRS-SO total scores can be organized into five risk levels using the common risk language (Hanson et al., 2016) corresponding to Very Low Level I (0-14.5), Below Average Level II (15-23.5), Average Level III (24-39.5), Above Average Level IVa (40-49.5), and Well Above Average Level IVb (50-72) (Olver, Mundt, et al., 2018). Alternatively, the Static-99R can be substituted in place of the VRS-SO static factors and integrated with the VRS-SO's dynamic items to generate risk cutoffs following the five-level scheme (Olver et al., 2019). In turn, VRS-SO scores with or without Static-99R are linked to logistic regression generated 5- or 10-year sexual or violent recidivism estimates by way of an online calculator (Mundt, 2015, www.psynergy.ca) that uses a logistic function to combine specific scores for static, dynamic, and change scores.

\section{Procedure and Plan of Analysis}

VRS-SO and Static-99R ratings had been completed on a nationwide prospective sample of men who had attended sexual violence reduction services through the NaSOP, including the Clearwater Program. The VRS-SO was administered as part of routine services pre- and posttreatment by psychology staff and/or program facilitators who were trained by one of the VRS-SO co-authors or designees experienced with the tool. VRS-SO data were entered electronically and archived at CSC National Headquarters (NHQ), stored in hardcopy format at NHQ, or entered electronically into a secure institutional repository (Clearwater Program only). The Static-99 was also rated by frontline staff and archived in these forums and later converted to Static-99R scores by reweighting the age item per scoring rules (Helmus et al., 2012). Following the coordination, entry, and integration of these data, DO designation was flagged when post-release outcome data were gathered from the Canadian Police Information Centre (CPIC). On these men's files and in their sentencing information, their DO status was clearly indicated as such. Not all men adjudicated DO necessarily had risk assessment information available from the data source. As such, the data examined represent all cases with risk and sexual offense treatment information.

The presentation and discussion of these data are organized along five propositions regarding the nature of the risk-need profile of DOs in Canadian correctional settings. 
To generate these findings, I conducted basic descriptive statistics for the risk-need profiles of the DO sample, compared these to other historically high risk-high need (HRHN) treated sexual offense samples, and used VRS-SO normative information and data interpretative tools to contextualize the men's risk-need profiles and ultimately the sexual recidivism risk of the sample as a whole.

\section{Results and Discussion}

\section{Proposition \#1: Treating, Reintegrating, and Releasing HRHN Cases Is "Business as Usual"}

Sexual offense treatment programs (SOTP) in Canadian corrections have a long history of treating HRHN men and releasing them back to the community without evidence of new sexual offenses occurring per the risk, need, responsivity (RNR) principles (Andrews $\&$ Bonta, 2010). This is nothing new. For this reason, Canada's federal correctional department assigns men to levels of treatment intensity commensurate with their level of risk, and specifically, has high intensity streams of mainstream programming, including SOTP, for HRHN cases. Indeed, research from the broader NaSOP VRS-SO sample demonstrated that high intensity program attendees had significantly higher Static-99R and VRS-SO scores, compared to men attending the moderate intensity stream, to those in the low intensity stream (Olver et al., 2014, 2020). As described earlier, the SOTP targets criminogenic needs through a series of group-based modules and individual services (need principle), using cognitive behavioral methods of change (general responsivity). Services and provisions are in place to modify delivery (or refer to programs with modified delivery) based on the men's cognitive capabilities and learning style, cultural heritage (e.g., Indigenous programming streams), motivation and the like (specific responsivity). Support and reintegration planning services are provided by treatment staff and parole officers within the institution and community. Additional community services and supports are provided through release planning (e.g., job, residence, family), maintenance programming, and/or other services to aid successful reintegration, such as Circles of Support and Accountability (CoSA). The result? As demonstrated in Table 1, the majority of HRHN men with a history of sexual offending released to the community do not return to custody for new sexual or even violent offenses.

Table 1 provides a breakdown of VRS-SO and Static-99R information for three sets of Canadian treated HRHN samples and their recidivism rates. The Clearwater I sample (Olver et al., 2007) featured all admissions from 1983 to 1997, prior to the formalization of the RNR principles, so the men may not have consistently been actuarially high risk, but they were so referred because they had other psychological concerns that warranted their referral to a high intensity residential program. The NaSOP high intensity and Clearwater II samples are more homogeneously high risk and so-referred based on their 


\section{Table 1}

VRS-SO/Static-99R Risk Profiles and Recidivism Rates From High Intensity Sexual Offense Treatment Programs in Canadian Corrections

\begin{tabular}{|c|c|c|c|c|c|c|}
\hline \multirow[b]{2}{*}{ Measure } & \multicolumn{2}{|c|}{$\begin{array}{c}\text { Olver et al., 2007, } \\
\text { Clearwater I } \\
(N=321)\end{array}$} & \multicolumn{2}{|c|}{$\begin{array}{l}\text { Olver et al., 2014, } \\
\text { 2020, National Sex } \\
\text { Offender Program } \\
\text { (High Intensity) } \\
(N=254)\end{array}$} & \multicolumn{2}{|c|}{$\begin{array}{c}\text { Sowden \& Olver, } \\
\text { 2017, Clearwater II } \\
(N=180)\end{array}$} \\
\hline & $M(S D)$ & $\%(n)$ & $M(S D)$ & $\%(n)$ & $M(S D)$ & $\%(n)$ \\
\hline \multicolumn{7}{|l|}{ Risk measure } \\
\hline Static-99R & $4.6(2.3)$ & - & $5.3(2.1)$ & - & $4.9(2.2)$ & - \\
\hline VRS-SO static & $10.0(4.0)$ & - & $11.4(3.5)$ & - & $11.1(3.6)$ & - \\
\hline VRS-SO dynamic pre & $25.0(7.5)$ & - & $31.8(6.7)$ & - & $31.2(5.4)$ & - \\
\hline VRS-SO dynamic post & $22.4(7.4)$ & - & $27.7(6.8)$ & - & $27.0(6.0)$ & - \\
\hline VRS-SO change & $2.6(2.1)$ & - & $4.0(2.9)$ & - & $4.2(3.3)$ & - \\
\hline VRS-SO pretreatment total & $34.9(10.0)$ & - & $43.3(8.5)$ & - & $42.3(7.4)$ & - \\
\hline VRS-SO posttreatment total & $32.4(9.9)$ & - & $39.2(8.5)$ & - & $38.2(7.6)$ & - \\
\hline \multicolumn{7}{|l|}{ Recidivism criterion } \\
\hline Sexual 5-year & - & 18.1 & - & 14.2 & - & 12.5 \\
\hline Sexual 10-year & - & 25.5 & - & 18.5 & - & 25.7 \\
\hline Violent 5-year & - & 34.4 & - & 28.0 & - & 32.7 \\
\hline Violent 10-year & - & 48.1 & - & 41.2 & - & 52.4 \\
\hline
\end{tabular}

Note. VRS-SO = Violence Risk Scale-Sexual Offense Version.

actuarial risk level. Several themes are evident from this table. First, Static-99R scores were in the Above Average risk (Level IVa) category overall (Hanson et al., 2017), as were the VRS-SO pretreatment dynamic and total scores (Olver, Mundt, et al., 2018) for the NaSOP and Clearwater II streams; Clearwater I has a larger SD to reflect the greater risk level variability. Second, across each sample, the men reduced their risk overall, amounting anywhere from one third (Olver et al., 2007) to approximately $0.50 \mathrm{SDs}$, for the two later programs. Accordingly, the VRS-SO numeric score and risk level has been reduced from IVa overall to the upper end of Level III for NaSOP and Clearwater II. Outcome research from each of these samples has demonstrated VRS-SO change scores to be significantly associated with reductions in sexual and/or violent recidivism, controlling for baseline pretreatment risk level. Finally, the samples had 5-year rates of sexual recidivism comparable to the Static-99R routine samples $(15.2 \%)$ and below that for the HRHN Static-99R sample (21.2\%) (Phenix et al., 2016). The 10-year observed rates of sexual recidivism (ranging from about 18-25\%) were also lower than the $32.1 \%$ 10-year 
rate for HRHN norms. As would be anticipated, the 5 and 10-year rates of violent (sexual and nonsexual) recidivism are higher, but even still, a majority of HRHN men do not return to custody for a new violent offense within these follow-up periods; those who reduce their risk have lower rates of return.

\section{Proposition \#2: Canadian DOs Have Similar Risk-Need Profiles to Other HRHN Cases}

Is there evidence to suggest that DOs are different than other HRHN men? From a risk and need perspective, specifically, scrutiny of the risk and need profiles of $33 \mathrm{DO}$ cases with VRS-SO and Static-99R information available demonstrate that they are not necessarily higher risk or greater need than other HRHN men who are not given a DO designation. The next set of comparisons focused on the NaSOP and Clearwater II samples, given that these men were referred on the basis of actuarial (Static-99R) score and post-dated the 1997 DO legislative developments that generated the indeterminate designation.

As seen in Table 2, the current sample of DO cases had a Static-99R score around 6 (Level IVb or Well Above Average Risk), and VRS-SO dynamic and total scores in the Above Average Risk (Level IVa) range, and that their dynamic scores had been reduced to Average Risk (Level III) posttreatment. These are de facto higher risk men; however, the dynamic scores at pre (range 31.2 to $31.8, d=0.02-0.09$ ) and posttreatment (range 27.0 to $27.8, d=0.02-0.13$ ) differed by less than 1 point between the DO cases with the NaSOP and Clearwater II samples, while total score differed by 1-2 points overall at pre ( 42.3 to $43.8, d=0.06$ to 0.19 ) and posttreatment ( 38.2 to $40.4, d=0.14$ to 0.28 ). The amount of change was indistinguishable between these three groups (range 4.0 to $4.2, d=0.03$ ). In short, the data indicate that Canadian DOs adjudicated for sexual offense are substantively the same population as other HRHN cases from a risk and need perspective, at least as assessed on the VRS-SO.

Table 2 further identifies that the DO sample had about 10 out of 17 dynamic items identified as criminogenic (i.e., risk relevant and meriting intervention). Thus, they have a large number of dynamic risk factors that warrant services, and in keeping with the Council of State Government guidelines (Hanson et al., 2016), appropriately require a high intensity program. Table 3 provides a synopsis of where the men placed on the three risk-need domains of the VRS-SO. Although each individual case has a different profile of risks and needs, at a broad aggregate level, these men were likely to be above average and have areas of concern across each of the three domains, reflecting problems with atypical sexual interests and sexual self-regulation, global antisociality (e.g., substance use, aggression, impulsivity), and attitudes and cognitions supportive of sexual offending and counterproductive to reintegration. 
Table 2

VRS-SO and Static-99R Risk-Need Profile for Canadian Dangerous Offender Sample

\begin{tabular}{lccccc}
\hline Measure & $\boldsymbol{n}$ & $\boldsymbol{M}(\boldsymbol{S D})$ & Range & CL Risk & Percentile \\
\hline Static 99-R & 33 & $5.8(1.8)$ & $2-9$ & IVb & 94.2 \\
VRS-SO & & & & \\
$\quad$ Static & 32 & $11.9(3.9)$ & $5-19$ & III & 71.5 \\
$\quad$ Dynamic pre & 33 & $31.7(7.2)$ & $15-44$ & IVa & 85.3 \\
Dynamic post & 26 & $27.8(7.4)$ & $13-40$ & III & 73.0 \\
Change & 26 & $4.1(3.0)$ & $0-9.5$ & N/A & 57.9 \\
$\quad$ Total pre & 32 & $43.8(9.8)$ & $26-60$ & IVa & 85.7 \\
$\quad$ Total post & 25 & $40.4(9.9)$ & $22-57$ & IVa & 75.7 \\
No. criminogenic needs & 33 & $10.4(3.1)$ & $4-15$ & IVa & - \\
\hline
\end{tabular}

Note. VRS-SO = Violence Risk Scale-Sexual Offense Version. VRS-SO Percentile information generated from VRS-SO Users' Workbook (Olver, Kelley, Johnson, \& Wong, 2019); Static-99R percentile obtained from 2016 Static-99R \& Static-2002R Evaluator's Workbook (Phenix, Helmus, \& Hanson, 2016). CL = common language.

Table 3

VRS-SO Criminogenic Need Information for Canadian Dangerous Offender Sample

\begin{tabular}{llccc}
\hline VRS-SO factor score & $\boldsymbol{n}$ & $\boldsymbol{M}(\boldsymbol{S D})$ & Range & Percentile \\
\hline Sexual deviance pre & 33 & $9.2(3.8)$ & $2-15$ & 70.3 \\
Sexual deviance post & 26 & $8.3(3.6)$ & $1.5-14$ & 62.2 \\
Criminality pre & 33 & $11.1(3.4)$ & $3-16$ & 77.2 \\
Criminality post & 26 & $9.7(3.0)$ & $3-15$ & 63.4 \\
Treatment responsivity pre & 33 & $7.3(2.7)$ & $2-12$ & 76.6 \\
Treatment responsivity post & 26 & $6.3(2.8)$ & $2-12$ & 64.0 \\
\hline
\end{tabular}

Note. VRS-SO = Violence Risk Scale-Sexual Offense Version. Percentile information generated from VRS-SO Users' Workbook (Olver, Kelley, Johnson, \& Wong, 2019).

It is worth noting from these two tables, however, that there were also actuarially (statically and dynamically) average to below average risk DOs in this sample with few treatment needs, which is a concern, given that the legislation in principle, is intended to manage risk and prevent recidivism, and is intended for only the most severe cases. For instance, of the 32 pretreatment VRS-SOs, 8 were Average risk (87.5\% White), 11 Above Average risk (81.8\% White), and 13 Well Above Average risk (53.8\% White). 


\section{Proposition \#3: Canadian DOs Can Make Risk Relevant Changes and Benefit From Risk Management Interventions}

The third proposition argues that even ostensibly high risk DO cases can make substantial risk relevant changes from SOTPs and concordant risk management and release planning. Given their similarity in risk and need profile to other HRHN men with sexual offense histories in Canadian corrections (and those specifically in high intensity SOTPs), this should hardly come as surprising. As seen in Table 4, three quarters of the DO sample were enrolled in a high intensity SOTP and about two thirds of men who attempted to complete their designated program (from those cases with this information available), successfully completed it. The rate of attrition (33\%) in this small sample is slightly higher than the $27 \%$ reported for SOTPs in the meta-analytic literature (Olver et al., 2011).

Table 4

Sexual Offense Treatment Program Variables, Change, and Projected Recidivism for Canadian Dangerous Offender Sample

\begin{tabular}{|c|c|c|c|}
\hline Variable & $n$ & $\%(n$ or $95 \% \mathrm{CI})$ & $M(S D)$, range \\
\hline Program intensity & 33 & & \\
\hline Moderate & & $24.2(8)$ & - \\
\hline High & & $75.8(25)$ & - \\
\hline Program length (mos) & 22 & - & $6.8(2.2), 4.0-12.2$ \\
\hline Successful completion & 21 & $66.7(14)$ & \\
\hline VRS-SO risk change & 26 & - & $\begin{aligned} & 4.1(3.0), 0-9.5 \\
d= & 0.58,57.9 \text { percentile }\end{aligned}$ \\
\hline \multicolumn{4}{|l|}{ Projected recidivism } \\
\hline Sexual 5-year & & $18.3[14.9,22.4]$ & - \\
\hline Sexual 10-year & & $28.3[24.5,32.4]$ & - \\
\hline Violent 5-year & & $38.3[33.9,42.9]$ & - \\
\hline Violent 10-year & & $52.4[46.9,57.8]$ & - \\
\hline
\end{tabular}

Note. VRS-SO = Violence Risk Scale-Sexual Offense Version. Percentile information generated from VRS-SO Users' Workbook (Olver, Kelley, Johnson, \& Wong, 2019). Recidivism projection based on VRS-SO normative sample $(N=913)$ calculator (Mundt, 2015) estimates with DO VRS-SO pretreatment $M=44.7$ and change $M=$ 4.1 .

As noted previously, the sample evinced the same level of change in their VRS-SO risk ratings from pre- to posttreatment as non-DOs from other high intensity programs; a potentially significant consideration, given that changes in sexual violence risk have 
been found to be associated with decreased sexual and violent recidivism (Olver, Mundt, et al., 2018). In the case of the current sample, this amounts to 4-points of change (i.e., or movement of a full stage on 8 treatment targets), or more than a one-half SD of change on dynamic risk factors over a mean program duration of approximately 7 months. The amount of change is also on par with the average amount of change registered in the VRS-SO normative sample, at around the $58^{\text {th }}$ percentile. In short, there is evidence that Canadian DO cases with a history of sexual offending can make meaningful reductions in risk associated with participation in evidence informed programming or other change agents.

\section{Proposition \#4: Canadian DO Recidivism Rates Are Not Necessarily Higher Than Other HRHN Cases}

The fourth proposition asserts that the prognosis for HRHN men who fare well in treatment, cooperate with their correctional plans, and actively engage in release planning, as shown by positive change in VRSSO scores, is quite positive and estimated recidivism rates are lowered. What would the 5- and 10-year sexual recidivism projections be for a typical HRHN DO who successfully completed an SOTP and registered the average amount of change as seen in the DO sample overall? I examined this through use of the VRS-SO calculator, inputting the average pretreatment total of 44.7 with 4.1 points of change, to generate 5- and 10-year logistic regression generated estimates of sexual and violent (i.e., sexual and nonsexual) recidivism (i.e., bearing in mind that none of these men had been released at the time of data capture). As seen in the bottom half of Table 4 , the rates of sexual and violent recidivism are consistent with the actual observed rates of the three treated HRHN samples reported in Table 1. Importantly, the lower bounds of the $95 \% \mathrm{CI}$ for the logistic regression generated estimates for the DO sample overlapped with the observed rates for one or more of the HRHN samples for each outcome; noting that all of the men in the HRHN samples were released. It is also important to remember that these are aggregate figures comparing entire samples. Certainly, men who make greater progress will have better reintegration prospects (and hence reduced potential for recidivism), than those men who fare poorly and make little changes to their risk. What this indicates is that the "typical" treated DO in the present sample does not appear to have substantively greater likelihood of future sexual or violent recidivism than other treated Canadian HRHN samples of men with a history of sexual offending. The kind of change shown in this instance has been associated with lower observed rates of recidivism in other samples, although projected reduced recidivism rates need to be verified with observed reduced rates. Future research optimally would examine: (a) whether VRS-SO scores are valid predictors of sexual recidivism for released DOs (e.g., discrimination metrics such as AUC and Cox regression), and (b) whether the VRS-SO norms (and calculator) apply to released DOs (e.g., calibration metrics such as expected/observed [E/O] analyses. 


\section{Proposition \#5: Dynamic Sexual Offense Risk Assessment Tools Have Utility With Canadian DO Cases and by Extension Possibly Other ID Sentenced Populations}

The fifth and final proposition posits that dynamic risk instruments can be used as repeated measures assessment tools (e.g., STABLE per Nunes et al., 2011; VRS-SO per Olver, Mundt, et al., 2018) to track progress and monitor risk change over multiple assessments to inform release and reintegration decisions with DOs or other ID cases (e.g., SVP). The VRS-SO as one example of a dynamic sexual violence risk assessment and intervention planning tool that serves these functions as illustrated by a hypothetical case in Figure $1 \mathrm{a} / 1 \mathrm{~b}$ and Figure $2 \mathrm{a} / 2 \mathrm{~b}$ to illustrate its use within an institutional setting. As seen in Figure 1a, per the risk principle, the VRS-SO can be used to identify the baseline risk level for future sexual violence to inform case prioritization and intensity of services. Per the need principle, the 17 dynamic items rated at baseline can be used to generate a criminogenic profile of the individual's risks and needs; specifically, key high-risk areas to be prioritized for services. The greater the volume and density of criminogenic need, the more pressing the need for high intensity as well as diverse and coordinated services. In this hypothetical example, items pertaining to general antisociality and attitudes and cognitions had the greatest treatment relevance, whereas this case had few identified concerns in areas of sexual deviance and regulation.

The VRS-SO also incorporates a framework termed offense analogue and offense replacement behaviors (OABs and ORBs), which can be formally assessed through the $\mathrm{OAB} / \mathrm{ORB}$ guide (Olver et al., 2017). OABs refer to offense linked proxy behaviors that represent a repackaging of a dynamic risk factor within a custodial environment where security and supervision could suppress offending behaviors. By contrast, ORBs are positive prosocial replacement behaviors that would indicate reduction of the risk factor and the generation of healthier alternatives (Olver \& Stockdale, 2020). For instance, a Sexually Deviant Lifestyle (D1) could be expressed through OABs such as sexually inappropriate behaviors or gestures toward staff or patients, exposure to triggering stimuli or media that is offense relevant (e.g., child images, sexually explicit narratives), or masturbation to deviant or inappropriate fantasy (e.g., past offenses, staff). Relevant ORBs for this item would include improved sexual self-regulation as evidenced by maintaining good sexual boundaries, avoidance of triggering stimuli, appropriate urge control (e.g., through mindfulness, distraction, of other prosocial outlets), and healthy sexual fantasy to cite but a few examples. The higher the rating on a given VRS-SO dynamic item, the greater the presence of prominent OABs and fewer ORBs. 


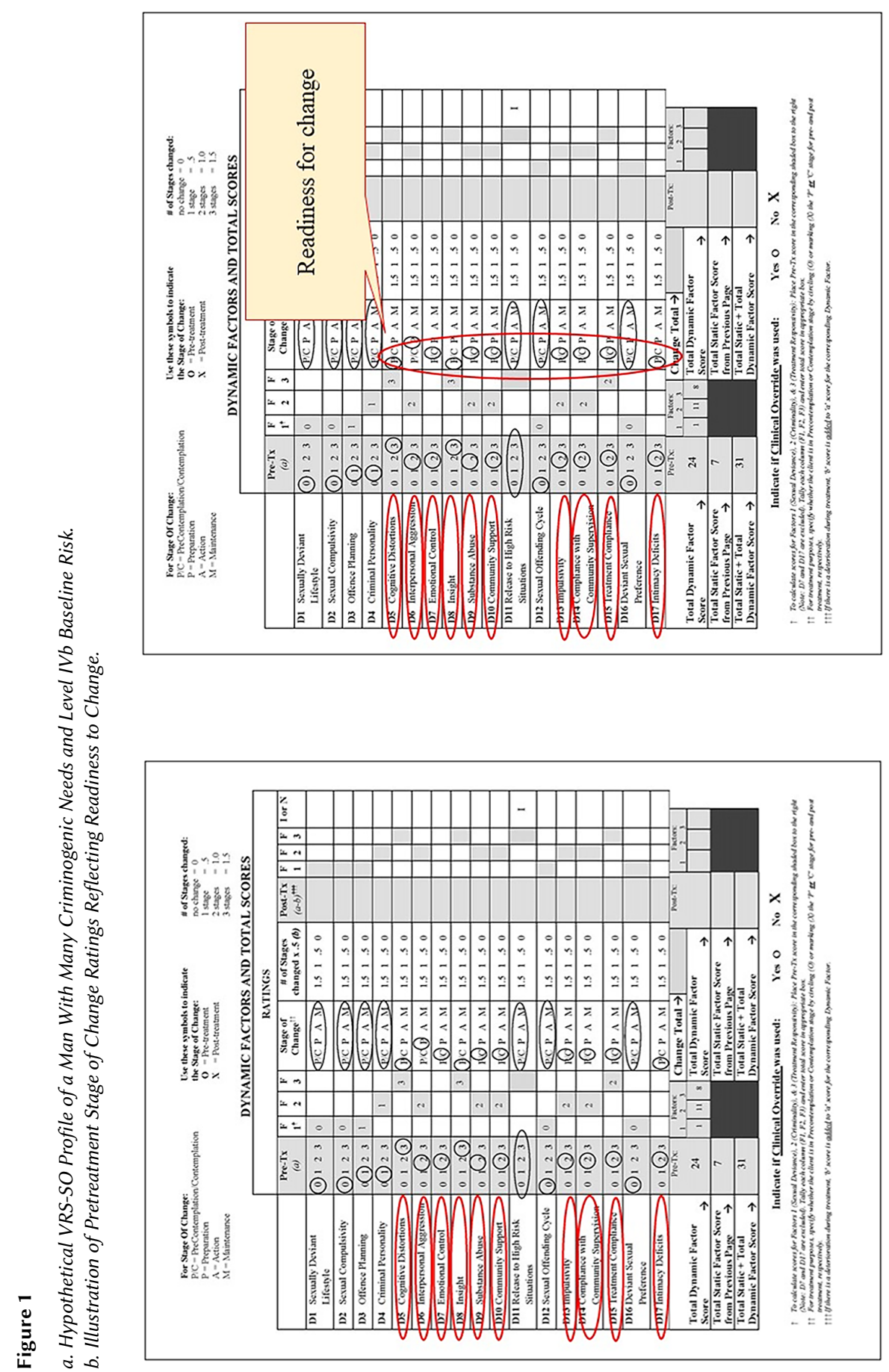

Sexual Offending: Theory, Research, and Prevention 2021, Vol. 16, Article e3745 


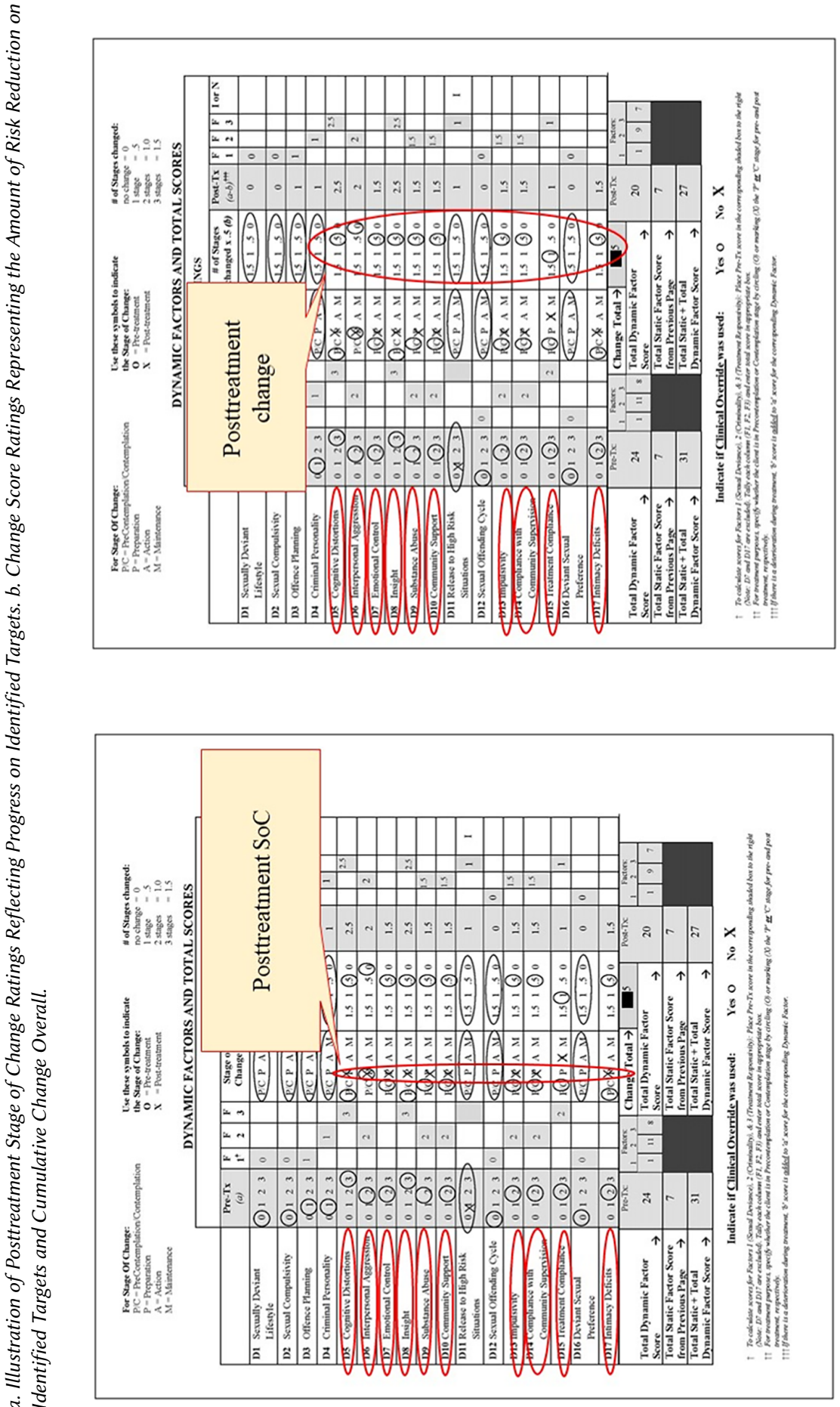

Sexual Offending: Theory, Research, and Prevention 
Figure 1b illustrates how the VRS-SO can be used to evaluate readiness for change using the stage of change model at baseline. Treatment interventions can be matched to the individual's readiness to change, per the responsivity principle. A string of precontemplation ratings would indicate an individual with little insight or awareness of his problem areas, or awareness of the issue, but little desire or intention to change. At such an early stage, stage matched interventions should be used to mobilize motivation (e.g., motivational interviewing) to increase client engagement and therapeutic buy in. The service provider can expect the client to be resistant to change efforts and perhaps even have an adversarial attitude toward treatment. In the case of Figure 1b, the client is primarily in contemplation and preparation on his treatment targets, demonstrating an awareness of and receptivity to making changes on a given domain (contemplation) in most areas; however, there clearly are areas in which the client is precontemplative (e.g., cognitions, insight, relationship concerns). The stage of change rating will also reflect the presence of OABs and ORBs; early stage of change ratings on criminogenic items would reflect the presence of prominent OABs and few or no ORBs. In the VRS-SO rating manual, the maintenance stage of change descriptors are a series of ORBs, while movement up the continuum of change is a reflection of the extent to which the individual demonstrates such behaviors, their frequency, stability, and longevity (Olver \& Stockdale, 2020).

Figure 2a illustrates progress across identified treatment targets, and how some areas may improve differentially compared to others. In this instance, areas not identified as problematic did not change, while in most substantive areas of risk and need, the client registered at preparation at posttreatment, demonstrating having begun the process of using risk reduction skills and strategies to manage a problem area; the changes are relatively recent and lapses may be frequent. Thus, these improvements in SoC reflect the presence of risk reduction behaviors and use of skills and strategies to manage a given risk factor. This also corresponds to an increase in ORBs (i.e., prosocial replacement behaviors) and deceased OABs; the more advanced the stage of change on a given item, and greater the number of cumulative changes across items, the greater the presence of $\mathrm{ORBs}$ to manage risk and maintain prosocial functioning. Accordingly, Figure $2 \mathrm{~b}$ shows how this may be quantified in terms of risk reduction on each of the VRS-SO's dynamic items. Most areas improved with gains that amounted to about a 0.5 -point decrease in score on a given item per stages of change of progress; the exception being 1.0 points (hence, two stages of change advanced) for sexual offense treatment compliance (D15).

Although the changes may appear to be small, they are cumulative across items and amount to a total of five points or approximately two-thirds of a standard deviation on the dynamic items; the change score represents the amount of risk reduction occurring to mitigate risk. The change ratings identify areas that may merit further attention through services and the overall change quantity in the end, relative to the individual's baseline risk can be used to generate a 5- or 10-year estimate for sexual or violent recidi- 
vism. As a repeated measures tool, the VRS-SO can be readministered at subsequent time points, with the previous administration serving as a new reference point or baseline and change tracked cumulatively over the total period of case management. In this manner, the VRS-SO can be used from the point of intake to case closure to guide reassessment and client progress toward reintegration.

\section{Conclusion}

In closing, ID sentenced men convicted for sexual offenses are not a novel entity in Canada's federal correctional department-government findings (Public Safety Canada, 2019) and reviews elsewhere (Nicholaichuk et al., 2013) suggest these men can be managed and safely reintegrated to the community. The findings from these data demonstrated that ID sentenced men have risk-need profiles that are similar to other HRHN men convicted for sexual offenses, that these men usually successfully complete sexual offense specific treatment and can and do make risk relevant changes. Estimates of future sexual reoffending over 5- and 10-year time frames as a function of risk, need, and change suggest that the rates are on par with other HRHN men that have been routinely released and successfully managed over the years by CSC. The VRS-SO as one example of a dynamic risk tool has particular relevance for an ID sentenced population given that the measure can be used to assess baseline risk, identify targets for treatment, and evaluate changes in risk to inform risk management efforts, including security reclassification decisions, release planning, supervision intensity, as well as areas for further intervention and monitoring. The use of the OAB/ORB framework (e.g., via the VRS-SO OAB/ORB guide) with the VRS-SO can augment these functions within institutional settings.

While the profile and risk projections for the ID-sentenced men in the current sample are informative and helpful, important study limitations are that these conclusions are based on a small sample with no actual release outcome and follow-up. Further research is needed on a larger sample to extend these findings, as well as a protracted time frame to examine observed (vs. projected) recidivism. These caveats aside, arguably dynamic assessment has the potential to help reintegration and can inform safe, fair, and humane decisions for ID sentenced persons. 
Funding: The author has no funding to report.

Acknowledgments: The author has no additional (i.e., non-financial) support to report.

Competing Interests: Mark Olver is one of the Editors-in-Chief of SOTRAP but played no editorial role for this particular article or intervened in any form in the peer review procedure.

Data Availability: The original data are not publicly available due to their highly sensitive and protected nature as well as the absence of formal operational or ethical approvals to provide this.

\section{References}

Andrews, D. A., \& Bonta, J. (2010). Rehabilitating criminal justice policy and practice. Psychology, Public Policy, and Law, 16, 39-55. https://doi.org/10.1037/a0018362

Beggs, S. M., \& Grace, R. C. (2010). Assessment of dynamic risk factors: An independent validation study of the Violence Risk Scale: Sexual Offender Version. Sexual Abuse, 22, 234-251. https://doi.org/10.1177/1079063210369014

Hanson, R. K., Babchishin, K. M., Helmus, L. M., Thornton, D., \& Phenix, A. (2017). Communicating the results of criterion referenced prediction measures: Risk categories for the Static-99R and Static-2002R sexual offender risk assessment tools. Psychological Assessment, 29, 582-597. https://doi.org/10.1037/pas0000371

Hanson, R. K., Bourgon, G., McGrath, R. J., Kroner, D., D’Amora, D. A., Thomas, S. S., \& Tavarez, L. P. (2016). A five-level risk and needs system: Maximizing assessment results in corrections through the development of a common language. Washington, DC, USA: Justice Center Council of State Governments.

Hanson, R. K., \& Thornton, D. (1999). Static 99: Improving actuarial risk assessments for sex offenders (User Report 99-02). Ottawa, Canada: Department of the Solicitor General of Canada.

Hanson, R. K., \& Thornton, D. (2000). Improving risk assessments for sex offenders: A comparison of three actuarial scales. Law and Human Behavior, 24, 119-136.

https://doi.org/10.1023/A:1005482921333

Helmus, L., Thornton, D., Hanson, R. K., \& Babchishin, K. M. (2012). Improving the predictive accuracy of Static-99 and Static-2002 with older sex offenders: Revised age weights. Sexual Abuse, 24, 64-101. https://doi.org/10.1177/1079063211409951

Kelley, S. M., Ambroziak, G., Thornton, D., \& Barahal, R. M. (2020). How do professionals assess sexual recidivism risk? An updated survey of practices. Sexual Abuse, 32, 3-29. https://doi.org/10.1177/1079063218800474

Mundt, J. C. (2015). VRS-SO calculator. Retrieved from https://psynergy.ca/

Nicholaichuk, T. P., Olver, M. E., Gu, D., \& Takahashi, Y. (2013). Correctional careers of dangerous offenders. Criminal Law Quarterly, 59, 487-497. 
Nunes, K. L., Babchishin, K. M., \& Cortoni, F. (2011). Measuring treatment change in sex offenders: Clinical and statistical significance. Criminal fustice and Behavior, 38, 157-173. https://doi.org/10.1177/0093854810391054

Olver, M. E., \& Eher, R. (2020). Predictive properties and factor structure of the VRS-SO: Results in an Austrian sample. European fournal of Psychological Assessment, 36, 748-757. https://doi.org/10.1027/1015-5759/a000551

Olver, M. E., Gordon, A., \& Wong, S. C. P. (2017). Offense analogue and offence replacement behavior $(\mathrm{OAB} / \mathrm{ORB})$ rating guide for Violence Risk Scale: Sexual Offense version. Available from mark.olver@usask.ca

Olver, M., Kelley, S., Johnson, L., \& Wong, S. (2019). Violence Risk Scale-Sexual Offense version (VRSSO): Users' workbook. Retrieved from www.psynergy.ca

Olver, M. E., Mundt, J. C., Thornton, D., Beggs Christofferson, S. M., Kingston, D. A., Sowden, J. N., Nicholaichuk, T. P., Gordon, A., \& Wong, S. C. P. (2018). Using the Violence Risk Scale-Sexual Offense Version in sexual violence risk assessments: Updated risk categories and recidivism estimates from a multisite sample of treated sexual offenders. Psychological Assessment, 30, 941-955. https://doi.org/10.1037/pas0000538

Olver, M. E., Neumann, C. S., Kingston, D. A., Nicholaichuk, T. P., \& Wong, S. C. P. (2018). Construct validity of the Violence Risk Scale-Sexual Offender version instrument in a multisite sample of treated sexual offenders. Assessment, 25, 40-55. https://doi.org/10.1177/1073191116643819

Olver, M. E., Nicholaichuk, T. P., Kingston, D. A., \& Wong, S. C. P. (2014). A multisite examination of sexual violence risk and therapeutic change. Journal of Consulting and Clinical Psychology, 82, 312-324. https://doi.org/10.1037/a0035340

Olver, M. E., Nicholaichuk, T. P., Kingston, D. K., \& Wong, S. C. P. (2020). A prospective multisite examination of dynamic sexual violence risk: An extension and update to Olver, Nicholaichuk, Kingston, and Wong (2014). Journal of Consulting and Clinical Psychology, 88, 362-371. https://doi.org/10.1037/ccp0000478

Olver, M. E., \& Stockdale, K. C. (2020). Assessing and managing offense analogue and offense replacement behaviors in correctional settings. Advancing Corrections, 10(3), 42-55.

Olver, M. E., Stockdale, K. C., \& Wormith, J. C. (2011). A meta-analysis of predictors of offender treatment attrition and its relationship to recidivism. Journal of Consulting and Clinical Psychology, 79, 6-21. https://doi.org/10.1037/a0022200

Olver, M. E., Wong, S. C. P., Nicholaichuk, T., \& Gordon, A. (2007). The validity and reliability of the Violence Risk Scale-Sexual Offender version: Assessing sex offender risk and evaluating therapeutic change. Psychological Assessment, 19, 318-329. https://doi.org/10.1037/1040-3590.19.3.318

Phenix, A., Helmus, L. M., \& Hanson, R. K. (2016, October 19). STATIC-99R \& STATIC-2002R evaluators' workbook. Retrieved from http://www.static99.org 
Prentky, R. A., Janus, E., Barbaree, H., Schwartz, B. K., \& Kafka, M. P. (2006). Sexually violent predators in the courtroom: Science on trial. Psychology, Public Policy, and Law, 12, 357-393. https://doi.org/10.1037/1076-8971.12.4.357

Prochaska, J. O., DiClemente, C. C., \& Norcross, J. C. (1992). In search of how people change: Applications to the addictive behaviors. The American Psychologist, 47, 1102-1114. https://doi.org/10.1037/0003-066X.47.9.1102

Public Safety Canada. (2019). 2018 annual report: Corrections and Conditional Release Statistical Overview. Sec. 753 (1-4), CCC. Dangerous offenders and long-term offenders. Retrieved from http://www.publicsafety.gc.ca/cnt/rsrcs/pblctns/ccrso-2018/index-en.aspx

Trevathan, S., Crutcher, N., \& Moore, J. (2002). A profile of federal offenders designated as dangerous offenders or serving long-term supervision orders (Research report No. R-125). Public Safety Canada, Corrections Research.

Wong, S., Olver, M. E., Nicholaichuk, T. P., \& Gordon, A. (2003-2017). The Violence Risk Scale: Sexual Offense version (VRS-SO). Saskatoon, Saskatchewan, Canada: Regional Psychiatric Centre and University of Saskatchewan.

Yates, P. M., Goguen, B. C., Nicholaichuk, T. P., Williams, S. M., \& Long, C. A. (2000). National Sex Offender Programs: Vol. 2. Moderate intensity. Ottawa, ON, Canada: Correctional Service of Canada.

IATSO

International Association for the
Ireatment of Sexual Offenders

Sexual Offending: Theory, Research, and Prevention (SOTRAP) is the official journal of the International Association for the Treatment of Sexual Offenders (IATSO).
(P) leibniz-psychology.org

PsychOpen GOLD is a publishing service by Leibniz Institute for Psychology (ZPID), Germany. 